\title{
Guidance and Control for Flat-Circular Parachutes
}

\author{
Scott Dellicker* \\ U.S. Army Yuma Proving Grounds, Yuma, Arizona 85365-9110 \\ Richard Benney ${ }^{\dagger}$ \\ U.S. Army Soldier and Biological Chemical Command, Natick, Massachusetts 01760-5017 \\ and \\ Glen Brown* \\ Vertigo, Inc., Lake Elsinore, California 92531-0117
}

\begin{abstract}
The Affordable Guided Airdrop System (AGAS) is being evaluated as a low-cost alternative for meeting the military's requirements for precision airdrop. Designed to bridge the gap between relatively expensive high-glide ratio parafoil systems and uncontrolled ballistic parachutes, the AGAS concept offers the benefits of high-altitude parachute releases as well as the potential for highly accurate point-of-use delivery of material. The design goal of the AGAS development is to provide a guidance, navigation, and control system that can be placed in line with cargo parachute systems, for example the G-12 flat-circular parachute, and standard delivery containers (A-22) without modifying these fielded systems. The AGAS is required to provide an accuracy of $328 \mathrm{ft}(100 \mathrm{~m})$, circular error probable (CEP), with a desired goal of $164 \mathrm{ft}(50 \mathrm{~m})$ CEP. The feasibility of this concept was investigated through modeling and simulation. A three-degree-of-freedom (3DOF) point mass flight dynamics model, sensor models of a commercial global positioning system (GPS) receiver and magnetic compass, and a model of the control and actuator system were incorporated into a Monte Carlo simulation tool. A bang-bang controller was implemented with trajectory tracking algorithms using position and heading information. Flight testing, using a radio-controlled scaled prototype, provided parachute dynamic and control response data to support the modeling efforts. The study demonstrated that this concept has the potential to provide control of previously unguided round parachutes to accuracies of approximately $210 \mathrm{ft}(64 \mathrm{~m}) \mathrm{CEP}$. The program is now continuing into the next phase to include the development of a full-scale prototype system for payloads up to $2200 \mathrm{lb}(1000 \mathrm{~kg})$.
\end{abstract}

$A$

${ }^{A}\left({ }^{C} P_{D}\right)$

$B$

$\{B\}$

$C_{D} S$

${ }^{C} F$

${ }^{C} N$

${ }^{C} P_{D}$

${ }_{A}^{C} R$

${ }^{C} V_{D}$

${ }^{C} \Omega_{D}$

$D$

$d$

$\mathrm{d} / \mathrm{d} t$

$I_{i i}$

$L, M, N$

\section{Nomenclature ${ }^{\S}$}

$=$ apparent mass matrix

$=$ position of point $D$, measured in $\{C\}$ and expressed in $\{A\}$ where

$$
{ }^{A}\left({ }^{C} P_{D}\right)={ }_{C}^{A} R\left({ }^{C} P_{D}\right) \neq{ }^{C} P_{D}
$$

$=$ body axis

$=$ name of the coordinate system

$=$ drag area

$=$ forces in $\{C\}$

$=$ moments in $\{C\}$

$=$ position of point $D$, measured in $\{C\}$ and expressed in $\{C\}$

$=$ rotation matrix from coordinate system $\{A\}$ to coordinate system $\{C\}$

$=$ velocity of point $D$, measured in $\{C\}$ and expressed in $\{C\}$

$=$ angular velocity of point $D$, measured in $\{C\}$ and expressed in $\{C\}$

$=$ drag

$=$ distance

$=$ time derivatives in the body-axis $\{B\}$

$=$ moment of inertia

$=$ moments in the $x, y, z$ axis, respectively

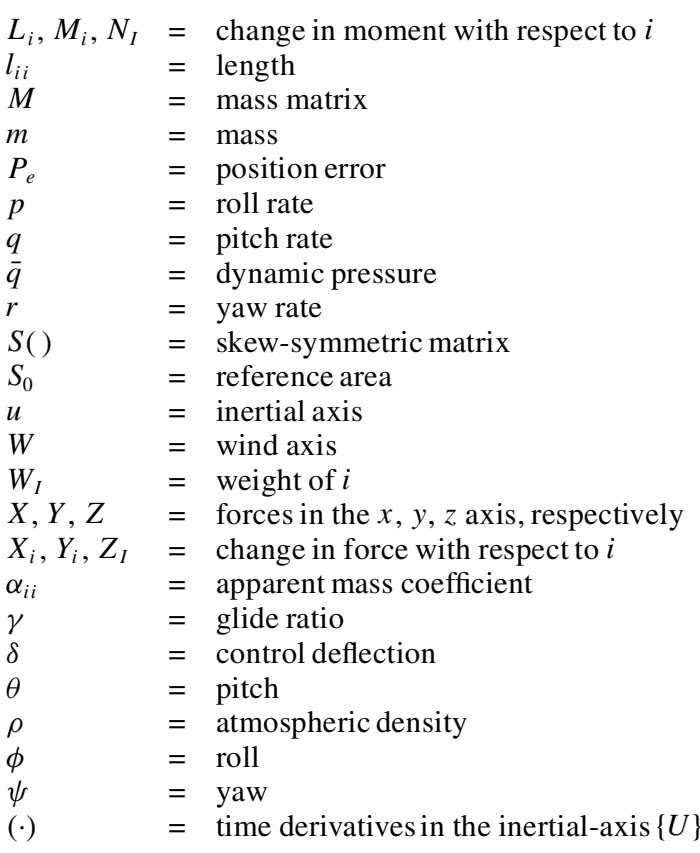

Subscripts

Received 20 February 2000; revision received 1 September 2000; accepted for publication 6 September 2000. This material is declared a work of the U.S. Government and is not subject to copyright protection in the United States.

* Chief, Aviation and Airdrop Systems Division, CSTE-DTC-YP-MT-EA.

†Ardrop Technology Team Leader, Natick Soldier Center.

President, P.O. Box 117.

${ }^{\S}$ Capital letters/symbols denote vectors or matrices. Small letters/symbols denote scalars. If coordinate axis symbol is omitted, $\{U\}$ is assumed.

$\begin{array}{ll}\text { AERO } & =\text { aerodynamic } \\ \text { AM } & =\text { apparent mass } \\ a & =\text { actuator } \\ c & =\text { canopy } \\ \text { c.g. } & =\text { center of gravity } \\ e & =\text { error } \\ \text { GRAV } & =\text { gravity } \\ p & =\text { payload } \\ x x \text { or } 1 & =x \text { axis }\end{array}$




$\begin{array}{ll}y y \text { or } 2 & =y \text { axis } \\ z z \text { or } 3 & =z \text { axis } \\ 1 & =\text { suspension lines }\end{array}$

\section{Introduction}

$\mathbf{T}$ HE U.S. Air Force Science Advisory board report titled "New World Vistas, Air and Space Power for the 21st Century"1 identified a critical need to improve the accuracy of airdrop of material. As a result of this study, a U.S. Army and U.S. Air Force team was formed to study precision airdrop. This team formulated the New World Vista, Precision Air Delivery program that seeks to demonstrate improved high-altitude cargo air delivery with a goal of achieving accuracies less than $164 \mathrm{ft}(50 \mathrm{~m})$ circular error probable (CEP). The program is investing in three areas: 1) improved wind estimation integrating mesoscale modeling with field wind measurements, 2) automated computed aerial release point (CARP) calculations using enhanced modeling of unguided parachutes, and 3) advanced decelerators.

To date, significant emphasis in the development of advanced decelerators has been placed on large-scale parafoil systems. These systems provide the accuracy required with delivery from high altitude and large offset distances. The drawback is relatively high cost for each pound of payload delivered. Alternate approaches were required to reduce system cost. The low-cost concept of the Affordable Guided Airdrop System ${ }^{2}$ (AGAS) is considered in this paper. This study encompassed modeling and simulation efforts to assess dynamic response of a flat circular parachute, to design guidance and control techniques, and to evaluate the feasibility of the AGAS concept.

\section{Agas Concept}

The design goal of the AGAS development is to provide a guidance, navigation, and control (GNC) system that can be placed in line with existing fielded cargo parachute systems (G-12) and standard delivery containers (A-22). The system is required to provide an accuracy of $328 \mathrm{ft}(100 \mathrm{~m}) \mathrm{CEP}$, with a design goal of $164 \mathrm{ft}(50 \mathrm{~m})$ CEP. The system should not require any changes to the parachute or cargo system.

The current design concept includes implementation of a commercial global positioning system (GPS) receiver and a magnetic compass as the navigation sensors, a guidance computer to determine and activate the desired control input, and the application of pneumatic muscle actuators (PMAs) ${ }^{3}$ to affect the control. The GNC system will be rigged with the payload, and the PMAs will go in line with each of four risers. Figure 1 illustrates this concept.

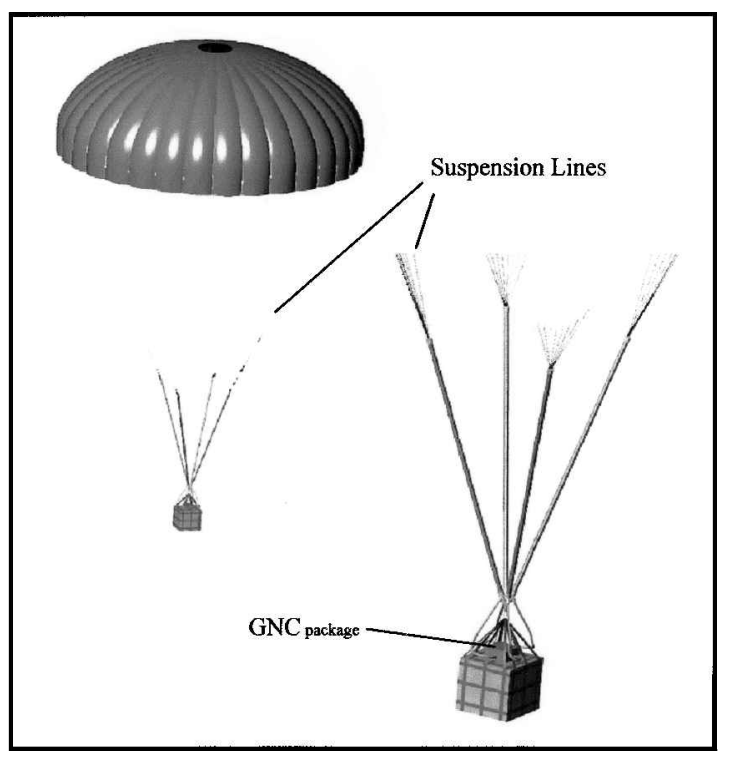

Fig. 1 AGAS concept illustration.

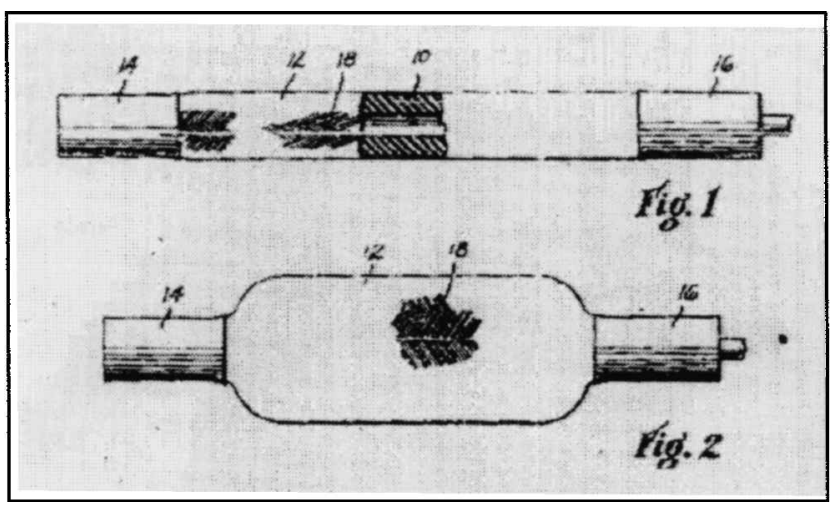

Fig. 21940 patent (Pierce, US, 2,211,478) drawing of pneumatic muscle.

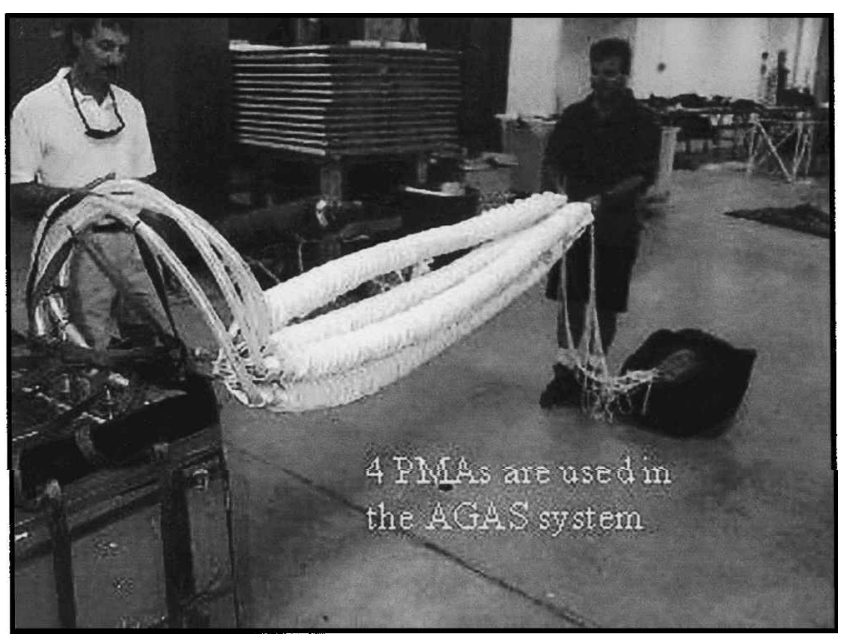

Fig. 3 Pneumatic muscle actuators.

\section{Pneumatic Muscle Actuators}

Vertigo, Inc., developed PMAs to affect the control inputs for this system. PMAs have been known, in concept, since at least 1940 when a similar device was shown in a patent document (Pierce, U.S. patent 2,211,478) for a mining application (Fig. 2).

A PMA is a braided-fiber tube that contracts in length and expands in diameter when pressurized. The contraction is quite forceful when compared to a piston-in-cylinderof the same diameter, and a contraction stroke of up to $40 \%$ of the original length is obtainable.

The PMA characteristics that were considered beneficial for AGAS were 1) efficient packing and reliable deployment, 2) high specific power, and 3) low manufacturing cost.

Uninflated PMAs, as installed on a scaled system, are shown in Fig. 3. Upon pressurization, the PMAs contract in length and expand in diameter. For this demonstration a displacement of approximately $3 \mathrm{ft}$ was selected. When depressurized, the PMAs are completely flexible allowing for efficient packing of the actuators with the parachute (Fig. 4). A reservoir of pressurized nitrogen is stored within the payload as the fuel source. The current fuel capacity allows for a maximum of 25 control inputs.

Initially, all actuators will be pressurizedupon successful deployment of the parachute. To affect control of the system, one or two actuators are depressurized, thereby lengthening one or two system risers. This action "deforms" the parachute (Fig. 5), creating drive in the opposite direction of the control action.

\section{Control System}

The accuracy of uncontrolled airdrop systems relies on precise knowledge of the winds at the time of the drop and precise guidance of the aircraft to the predicted release point. However, wind estimation is far from a precise science. The calculation of the CARP relies on less-than-perfect estimates of parachute aerodynamics, and the 


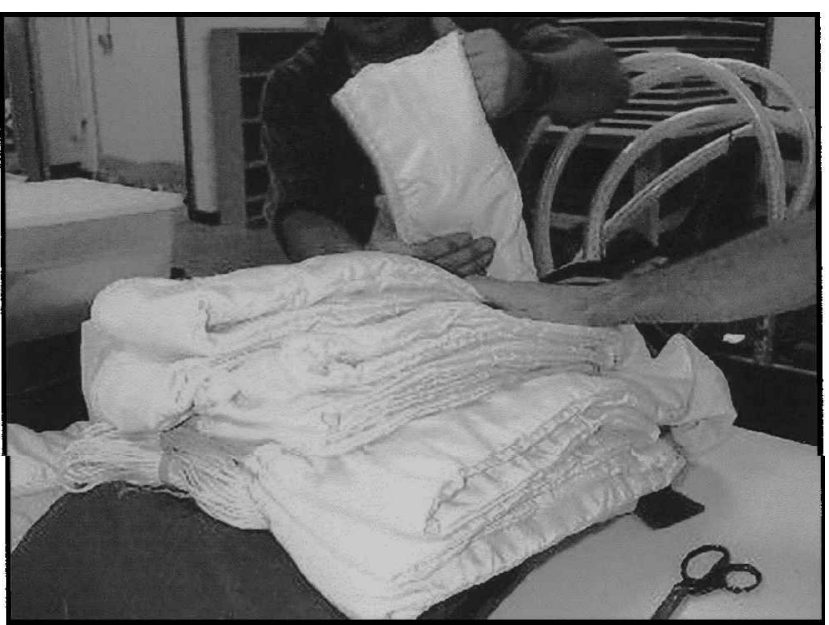

Fig. 4 Packing the parachute and actuators.

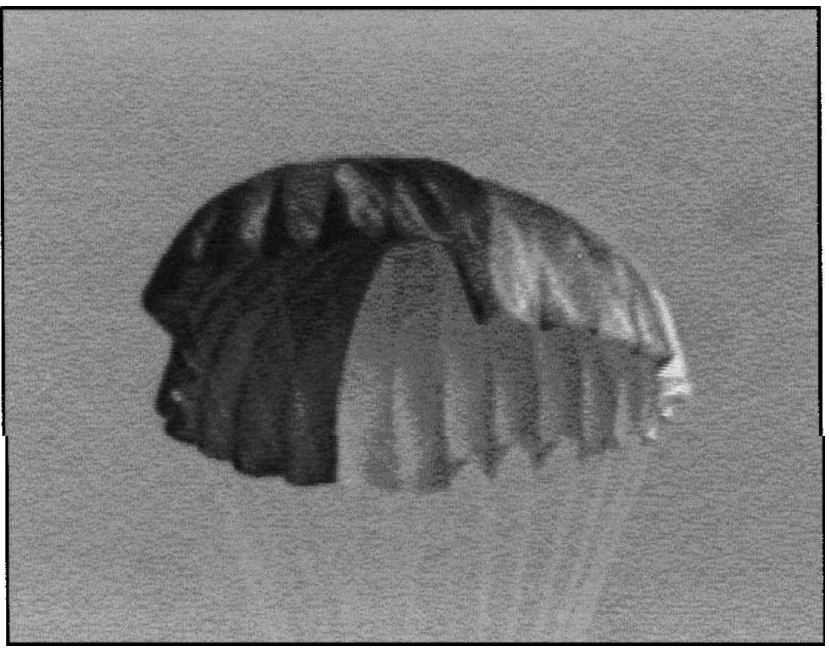

Fig. 5 Parachute with control activation.

aircraft crews cannot fly exactly to the predicted release point for each airdrop mission. Therefore, the AGAS control system design must help overcome these potential errors.

The parachutes to be used for this effort were not designed for glide or to be controlled. Therefore, limited control authority was expected. The G-12 parachute system is a flat-circular parachute (one, when lying flat on the ground, forms a circle) without any glide or control capabilities. A smaller flat-circular parachute, the C-9, was used for initial flight tests. This parachute was selected for this study as it has similar construction to the G-12 but is less than one-half the size, thereby simplifying test operations. Considering the relatively low glide ratio and a descent rate of approximately $25 \mathrm{ft} / \mathrm{s}(7.6 \mathrm{~m} / \mathrm{s})$, it is estimated the AGAS can overcome only a $12 \mathrm{ft} / \mathrm{s}(3.7 \mathrm{~m} / \mathrm{s})$ (approximately $7 \mathrm{kn}$ ) horizontal wind. It is therefore imperative to implement the system to overcome poor estimates in the wind and not try to steer the system against the entire wind. In other words, the drive of the system is insufficient to attempt to fly straight to the target but is likely sufficient to overcome errors in the wind estimate. For this reason, trajectory tracking techniques were selected. A preplanned trajectory, based on the best wind estimate available, must be determined and provided to the guidance computer. The GPS navigation system will provide continuous position of the system. The guidance computer will compare the actual horizontal position, at the system's current altitude, to the planned trajectory. This represents the position error $P_{e}$ at the current time. A tolerance cone is established about the planned trajectory (Fig. 6) starting at $600 \mathrm{ft}$ $(183 \mathrm{~m})$ at the beginning of the trajectory and gradually decreasing to $60 \mathrm{ft}(18.3 \mathrm{~m})$ at ground level. Should the position error be outside this tolerance, a control is activated to steer the system back

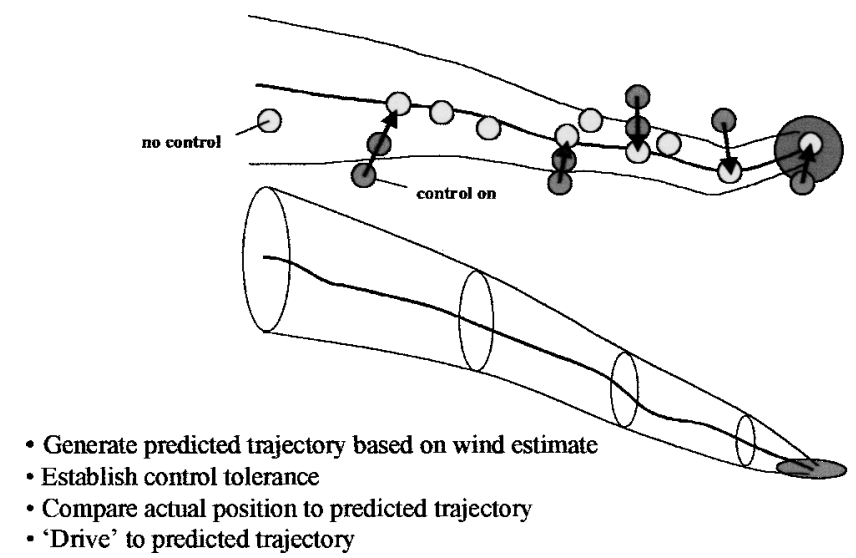

Fig. 6 Control concept.

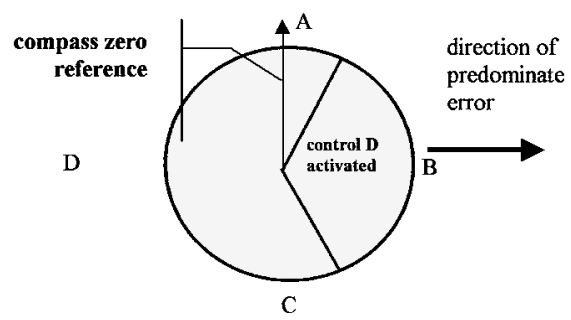

Fig. 7 Control activation.

to the planned trajectory. When the system is within $30 \mathrm{ft}(9.1 \mathrm{~m})$ of the planned trajectory, the control is disabled, and the parachute drifts with the wind. Thirty feet was selected to encompass approximately $1-\sigma$ of the GPS errors (each axis, no selective availability GPS errors).

As just outlined, the control system relies on the currenthorizontal position error to determine if control input is required. This position error $P_{e}$ is determined in inertial space and is then rotated to the body axis using an Euler angle rotation with heading only [Eq. (1)].

$$
P_{b}={ }_{u}^{b} R \cdot P_{e}
$$

where ${ }_{u}^{b} R$ is the Euler rotation matrix.

The resultant body-axis error $P_{b}$ is then used to identify which control input must be activated as shown in Eq. (2).

$$
\text { input }=\operatorname{sign}\left(P_{b} /\left\|P_{b}\right\|\right)
$$

Two components are returned, $\mathrm{a}+$ or - for the $x$ axis and $\mathrm{a}+$ or - for the $y$ axis. It was assumed for this simulation that $+x$ would activate control $A,-x$ activates control $C,+y$ activates control $B$, while $-y$ activates control $D$ (Fig. 7). The actual rigging of the operationalsystem must align these control actuators to the compass reference line to ensure proper control. Control $A$ is assumed to be aligned with the compass zero reference line.

The magnitude of the individual $x$ and $y$ components of the normalized body-axis position error vector is used to determine if the selected control will be activated. If the magnitude is greater than 0.3 , then that control is activated. This concept will allow the activation of a single control input or two simultaneous control inputs.

\section{System Modeling}

Three major components are included in the overall system model: 1) dynamics model, 2) sensor model, and 3) control system model. In addition, the reference trajectory generator was implemented using the same equations of motion used in the dynamics model.

\section{Dynamics Model}

In the absence of sufficient wind-tunneldata for the C-9 parachute, a point-mass system was assumed with the only forces on the system being drag and weight. Included in the weight are the effects 


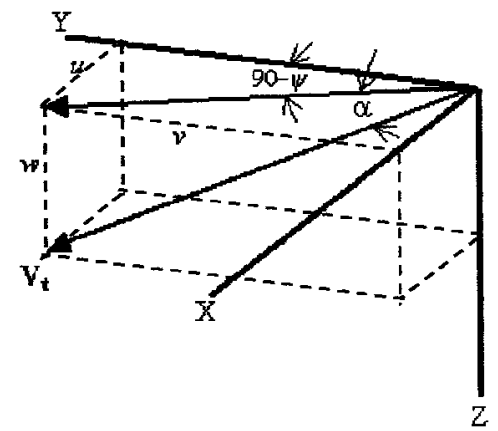

Fig. 8 Coordinate axes.

of apparent mass. As a body accelerates through a fluid, the fluid itself must accelerate to accommodate the motion of the body. Resultant forces and moments are applied to the body. A common method for accounting for these forces is to include added or apparent mass terms in the equations of motion. Sir Horace Lamb performed the original work on the effects of accelerating fluid on a body. ${ }^{4}$ Lamb derives the apparent mass effects and identifies 15 independent terms. Doherr and Saliaris ${ }^{5}$ demonstrated that for an equivalent parachute, four independent apparent mass terms exist considering six degrees of freedom. For the three-degree of freedom point-mass estimate, the following terms are considered:

$$
\alpha_{33}=\left\{4 / 3 \pi\left(D_{P} / 2\right)^{3}\right\}
$$

where $D_{p}$ is the profile diameter of the parachute.

$$
\alpha_{11}=\alpha_{22}=\frac{1}{2} \alpha_{33}
$$

Inherent in the point-mass estimate is that the coordinate axis of the system is aligned with the inertial axis, assuming a flat nonrotating Earth. This assumption places limits on the applicability of this model to an oscillating parachute system. Research ${ }^{5}$ has demonstrated that the aerodynamic forces of parachute systems can vary greatly with angle of attack. This model will not capture those dynamics. However, insufficient data on this parachute are available to formulate a full six-degree-of-freedommodel at this time. Figure 8 presents the coordinate axes used and defines the parameters in the equations of motion.

The derivation of the equations of motion for the uncontrolled parachute follows. Noting that:

$$
V_{G}=V_{A}+V_{W}
$$

where $V_{G}$ is the ground velocity, $V_{A}$ is velocity relative to the local air mass (airspeed), and $V_{W}$ is wind velocity. It assumes no rotation between the fixed Earth reference and the system's body axis.

By Newton's Law,

$$
M \dot{V}_{G}=F_{\mathrm{AERO}}+F_{\mathrm{GRAVITY}}
$$

The aerodynamic forces, in their individual components, are

$$
\begin{gathered}
X_{\mathrm{AERO}}=\left(m+\alpha_{11}\right) \dot{u}=-D \cos \gamma \cos \psi \\
Y_{\mathrm{AERO}}=\left(m+\alpha_{22}\right) \dot{v}=-D \cos \gamma \sin \psi \\
Z_{\mathrm{AERO}}=\left(m+\alpha_{33}\right) \dot{w}=-D \sin \gamma
\end{gathered}
$$

where $\gamma$ and $\psi$ are the flight-path angle and yaw angle, respectively.

$$
D=\bar{q} C_{D} S
$$

The gravitional forces are

$$
\begin{gathered}
X_{\mathrm{GRAV}}=0 \\
Y_{\mathrm{GRAV}}=0 \\
Z_{\mathrm{GRAV}}=W
\end{gathered}
$$

where $W$ is the system weight.
From geometry the reference angles are

$$
\begin{gathered}
\sin \gamma=w / V_{T}, \quad \cos \gamma=\sqrt{V_{T}^{2}-w^{2}} / V_{T} \\
\sin \psi=v / \sqrt{V_{T}^{2}-w^{2}}, \quad \cos \psi=u / \sqrt{V_{T}^{2}-w^{2}}
\end{gathered}
$$

Substituting, rearranging terms, and putting in state-space form

$$
\begin{gathered}
{\left[\begin{array}{c}
\dot{u} \\
\dot{v} \\
\dot{w}
\end{array}\right]=\left[\begin{array}{ccc}
m+\alpha_{11} & 0 & 0 \\
0 & m+\alpha_{22} & 0 \\
0 & 0 & m+\alpha_{33}
\end{array}\right]^{-1}} \\
\times\left\{\frac{-q C_{D} S}{V_{T}}\left[\begin{array}{c}
u \\
v \\
w
\end{array}\right]+\left[\begin{array}{c}
0 \\
0 \\
W
\end{array}\right]\right\} \\
\dot{V}_{G}=\dot{V}_{A}+\dot{V}_{W}
\end{gathered}
$$

Substituting, the Equations of Motion (EOM) for estimating ground speed results.

$$
\begin{aligned}
\dot{V}_{G}= & {\left[\begin{array}{c}
\dot{u} \\
\dot{v} \\
\dot{w}
\end{array}\right]_{G}=\left[\begin{array}{ccc}
m+\alpha_{11} & 0 & 0 \\
0 & m+\alpha_{22} & 0 \\
0 & 0 & m+\alpha_{33}
\end{array}\right]^{-1} } \\
& \times\left\{\frac{-q C_{D} S}{V_{T}}\left[\begin{array}{c}
u \\
v \\
w
\end{array}\right]+\left[\begin{array}{c}
0 \\
0 \\
W
\end{array}\right]\right\}+\dot{V}_{W}
\end{aligned}
$$

Adding in the control forces, we get the full set of equations of motion:

$$
\begin{aligned}
\dot{V}_{G}= & {\left[\begin{array}{ccc}
m+\alpha_{11} & 0 & 0 \\
0 & m+\alpha_{22} & 0 \\
0 & 0 & m+\alpha_{33}
\end{array}\right]^{-1} } \\
& \times\left\{\frac{-q C_{D} S}{V_{T}}\left[\begin{array}{c}
u \\
v \\
w
\end{array}\right]+\left[\begin{array}{c}
0 \\
0 \\
W
\end{array}\right]+\left[\begin{array}{c}
F_{\delta x} \\
F_{\delta y} \\
F_{\delta z}
\end{array}\right]\right\}+\dot{V}_{W}
\end{aligned}
$$

This set of equations can be solved numerically to estimate the system response. To estimate heading, a constant rate of rotation was assumed. The flight-test results for the C-9 parachute showed significant rotations and changes in rotation rate with control activation. Flight testing of the larger G-12 parachute showed a mean rotation rate of approximately $1.8 \mathrm{deg} / \mathrm{s}$. A standard deviation of $1.0 \mathrm{deg} / \mathrm{s}$ was assumed based on qualitative observations during flight test. An insufficient sample size was available to accurately determine a standard deviation for rotation rate. A normal random number generator at the start of each simulation determines the rotation rate. That rate is then integrated to provide heading. Multiple wind profiles were incorporated into the simulation using a look-up table based on the current system altitude.

\section{Sensor Models}

The AGAS is expected to include two navigation sensors: 1) a commercial GPS receiver for position determination and 2) a heading reference assumed to be a magnetic compass for this study. To assess the effects of navigation sensor errors, models for each sensor were be incorporated into the simulation.

GPS error sources include errors induced by the atmosphere (ionospheric and tropospheric), multipath, receiver noise, satellite clock noise, and selective availability. Selective availability is a means of intentionally inducing errors into the GPS satellite signal. The U.S. Department of Defense induces these errors to restrict use of the full precision of GPS to unauthorizedusers. Authorized users must apply a receiver capable of processing the cryptographiccodes to remove these induced errors. Although the AGAS concept could incorporate an authorized receiver, it is desired to utilize a commercial GPS receiver for cost savings. Also, with airdrop the loads may 


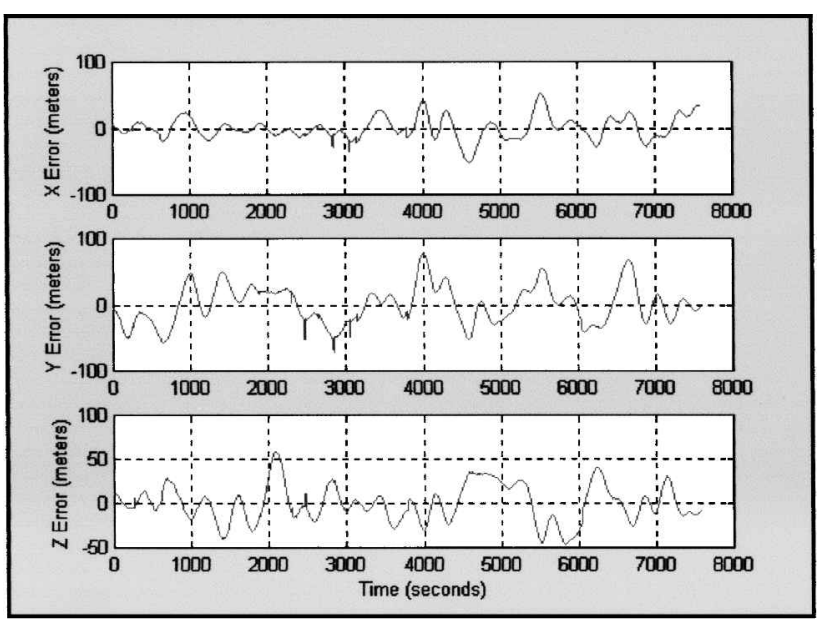

Fig. 9 Measured unauthorized GPS errors.

not be fully recoverable, and loss of the authorized receivers would not be desirable. Modeling techniques for GPS range errors resulting from these sources have been developed and validated ${ }^{6}$ to model range errors and not errors in a Cartesian reference as desired here. Cartesian $(\mathrm{x}, \mathrm{y}, \mathrm{z})$ errors would therefore have to be formed from the range errors for implementation in this simulation. This necessitates application of a numerical solution like maximum likelihood techniques. Although this implementation is relatively trivial, the computation resources required severely limit the simulation speed on a PC. Therefore, a variation of this approach was implemented.

The errors resulting from selective availability are not stochastic in nature. Therefore system identification methods were employed to obtain a reasonable error model. Data were collected at the Yuma Proving Ground Satellite Reference Station. An unauthorized GPS receiverwas placed on a known survey point. GPS position data were collected for over two hours. The position data in the three Cartesian axes were differenced with the surveyed coordinates resulting in the Cartesian errors. These errors represent all GPS error sources just identified. Figure 9 illustrates the apparent random nature of these data.

To obtain a model of these data, the MATLAB ${ }^{\circledR}$ system identification toolbox was utilized. An ARMAX ${ }^{8}$ model was used with the input being white noise and the output being the position errors just shown. The ARMAX model incorporates a prediction error method with a model represented by a set of difference equations of the form:

$$
A(q) y(t)=B(q) u(t-n k)+C(q) e(t)
$$

where $y$ and $u$ are the outputs and inputs of the system, respectively.

The coefficients $A, B$, and $C$ are polynomials that describe the model's difference equations. The prediction error is minimized using an iterative Gauss-Newton algorithm. The ARMAX function returns a matrix of the polynomial coefficients. This matrix, referred to as THETA format, can then be transformedinto a transferfunction using the MATLAB ${ }^{\circledR}$ command TH2TF. This technique resulted in the following transfer function that was used in the overall system model to obtain GPS errors:

$$
\frac{z^{4}-1.5302 z^{3}+0.2608 z^{2}+0.2566 z+0.0192}{z^{4}-2.6500 z^{3}+1.9582 z^{2}+0.0337 z-0.3420}
$$

Figure 10 presents the output of the GPS error model including selective availability errors.

The transfer function input is white noise initiated with a random seed ensuring variable errors are introduced from simulation to simulation. To assess the adequacy of this model, the mean, standard deviation, and rms were calculated for the measured and modeled GPS errors. The sample of measured errors just presented has a mean
Table 1 GPS position error model

\begin{tabular}{lcc}
\hline \hline Parameter & $\begin{array}{c}\text { Draper P-code } \\
\text { model }\end{array}$ & $\begin{array}{c}\text { C/A-code } \\
\text { model }\end{array}$ \\
\hline Accuracy standard deviation, $\mathrm{ft}$ & 30 & 45 \\
Accuracy correlation time constant, s & 0.1 & 0.1 \\
Jitter standard deviation, $\mathrm{ft}$ & 5 & 8 \\
Jitter correlation time constant, $\mathrm{s}$ & 0.05 & 0.05 \\
Uniform uncorrelated noise & & \\
$\quad$ standard deviation & $q /[2 * \operatorname{sqr}(3)]$ & $q /[2 * \operatorname{sqrt}(3)]$ \\
\hline \hline
\end{tabular}

$\overline{q \text { is the quantization interval. }}$

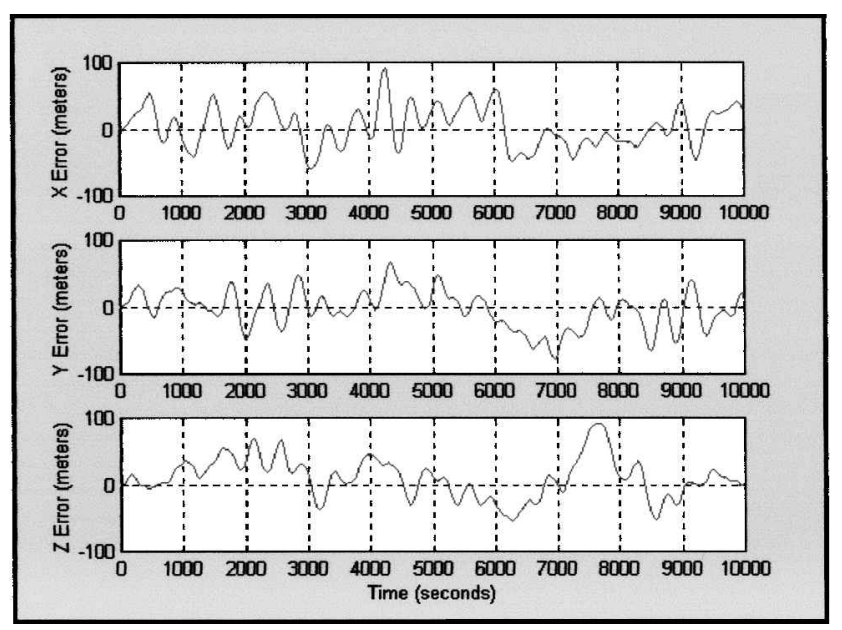

Fig. 10 Modeled GPS (unauthorized) errors.

value of approximately $0 \mathrm{ft}$ in each axis and a standard deviation of 56,94 , and $69 \mathrm{ft}(17,29$, and $21 \mathrm{~m})$ in the $x, y$, and $z$ axes, respectively. The modeled results demonstrated mean errors of 10 to $20 \mathrm{ft}$ ( 3 to $6 \mathrm{~m}$ ) with standard deviations ranging from 82 to $115 \mathrm{ft}$ ( 25 to $35 \mathrm{~m}$ ). The rms errors for the model were found to range from 85 to $122 \mathrm{ft}$ ( 26 to $37 \mathrm{~m}$ ) for three independent simulations. The model produces a reasonable representation of the measured GPS data.

With selective availability turned off, that is, no induced errors, a commercial GPS receiveris capable of navigating with greater accuracy. A GPS error model was derived considering a noise structure proposed by Draper Laboratory. ${ }^{9}$ This report models a P-code GPS receiver incorporated into the Honeywell Embedded GPS/Inertial Navigation System. The noise model incorporates two components: accuracy and jitter. The accuracy noise component is consideredexponentially correlated noise. The jitter component consists of two elements: an exponentiallycorrelated noise component with a faster time constant than the accuracy component and a uniform uncorrelated noise component. The GPS position noise model, suggestedfor GPS-only operations (no inertial aiding), was adapted for a commercial grade (C/A-code) receiver by adjusting the accuracy and jitter standard deviation specification. Table 1 presents the original and adapted models.

This model was incorporated into the Simulink ${ }^{\circledR}$ simulation. Figure 11 illustrates the results obtained from this model for a 50-s simulation. The standard deviation of the three-axis error for this simulation was $56.3 \mathrm{ft}(17.2 \mathrm{~m})$, which is close to the specifications for an commercial receiver with selective availability off.

The heading sensor is assumed to be a magnetic compass for this study. Two components of errors are considered here: a static error or bias and a dynamic (noise) component. System specifications for the attitude heading reference system (AHRS) provide a static error of $\pm 2 \mathrm{deg}$ ( $\pm 1 \mathrm{deg}$ with velocity aiding) and a dynamic component of $\pm 2 \%$. The AHRS incorporates rate gyros to obtain three-axis attitude rates and attitude data. Specification sheets of a low-cost digital magnetic compass produced by KVH Industries presented similar accuracy statements. The static error is incorporated as a bias element in the Simulink ${ }^{\circledR}$ model and is set as a uniform random variable at the start of each simulation. The dynamic component 


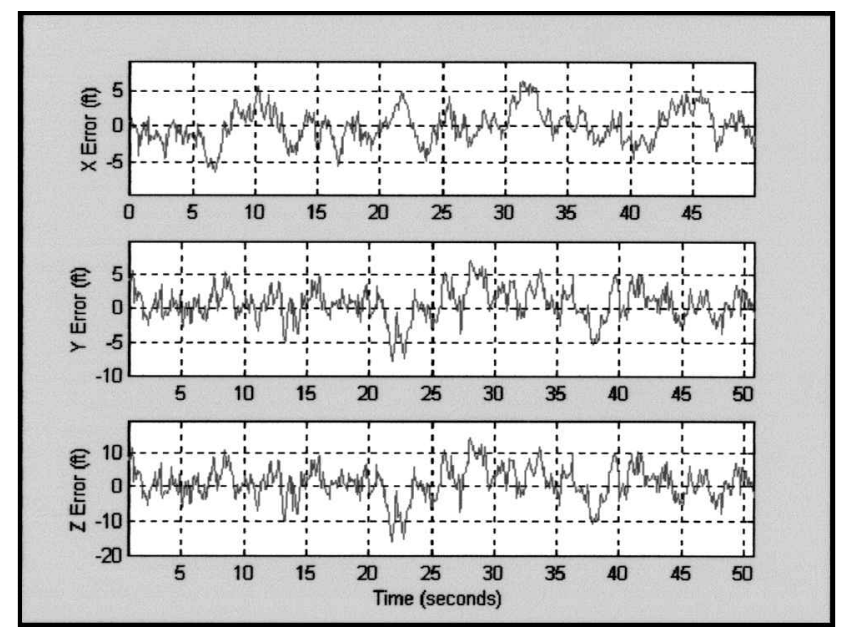

Fig. 11 GPS error model-selective availability off (50-s simulation).

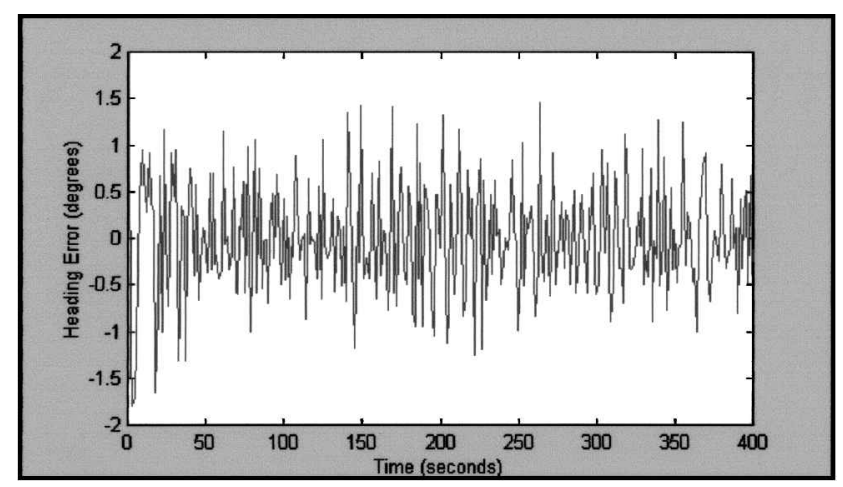

Fig. 12 Modeled heading error.

is found by adding $2 \%$ of the current heading reading. Figure 12 presents a 400 -s simulation of the heading error.

\section{Flight-Test Overview}

The flight-test effort focused on the collection of flight dynamic data to support modeling of the AGAS concept and was conducted with four actuators in line with a C-9 parachute (a $28-\mathrm{ft}(8.5 \mathrm{~m})$, flat-circular parachute) and a one-half scale container delivery system [300-lb (137 kg) payload]. The actuators were activated using a manual radio control system. Six-degree-of-freedomlight dynamic data were obtained including the position, velocity, acceleration, attitude, and attitude rates of the system. It was necessary to correlate these data with control inputs. Therefore, the state of control activation was also monitored. In addition, preliminary drop tests of the G-12 parachute (a $64-\mathrm{ft}(17 \mathrm{~m})$, flat-circular parachute) were conducted to assess qualitatively the differences in performance from that of the C-9 system and estimate effective PMA lengths, which are sized to maximize glide ratio.

\section{Instrumentation}

Ideally, both the parachute and payload would have been instrumented to collect all necessary data. However, the state of the art in instrumentation is not yet sufficient to instrument the parachute itself. As a result, only the payload could be instrumented. An instrumentation system (Fig. 13) was developed and included a differential GPS system for precise position and velocity, three-axis accelerometers for acceleration, and an AHRS for three-axis attitudes and attitude rates. Pressure transducers were put in line with the pneumatic actuators to monitor their action.

The accuracies of the measured data are presented in Table 2. These data are the compilation of the measurement sensors, $\mathrm{A} / \mathrm{D}$ conversion, and resultant resolution of the data storage and processing.
Table 2 Flight-test data accuracies/resolutions

\begin{tabular}{|c|c|c|c|}
\hline Parameter & Accuracy & Resolution & Sensor range \\
\hline GPS p & $\pm 3 \mathrm{~m}^{\mathrm{a}}$ & $\pm 0.1 \mathrm{~m}$ & Dynami \\
\hline GPS velocity & $\pm 0.1 \mathrm{~m} / \mathrm{s}^{\mathrm{a}}$ & $\pm 0.01 \mathrm{~m} / \mathrm{s}$ & Dynamics up to $4 g$ \\
\hline Three-axis attitude & $\pm 0.5 \mathrm{deg}^{\mathrm{a}}$ & $\pm 0.1 \mathrm{deg}$ & $\pm 90 \mathrm{deg}$ \\
\hline & & & $0-360$ deg heading \\
\hline Three-axis attitude rates & $\pm 0.1 \mathrm{deg} / \mathrm{s}^{\mathrm{b}}$ & $\pm 0.01 \mathrm{deg} / \mathrm{s}$ & $\pm 100 \mathrm{deg} / \mathrm{s}$ \\
\hline Three-axis acceleration & $\pm 0.1 g^{\mathrm{b}}$ & $\pm 0.01 \mathrm{~g}$ & $0-10 \mathrm{~g}$ \\
\hline Actuator pressure & $\pm 1 \mathrm{psi}^{\mathrm{b}}$ & $\pm 0.1 \mathrm{psi}$ & $0-250 \mathrm{psi}$ \\
\hline
\end{tabular}

${ }^{a}$ Measured against other comparable range instrumentation systems.

${ }^{\mathrm{b}}$ Obtained from manufacturer specifications.

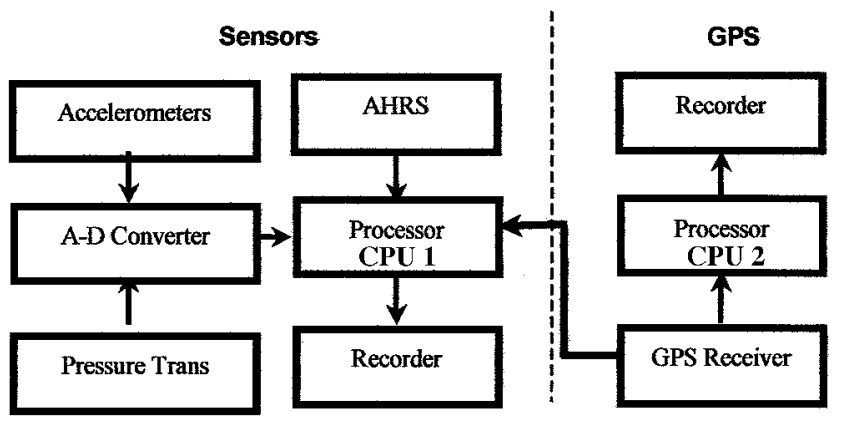

Fig. 13 Instrumentation block diagram.

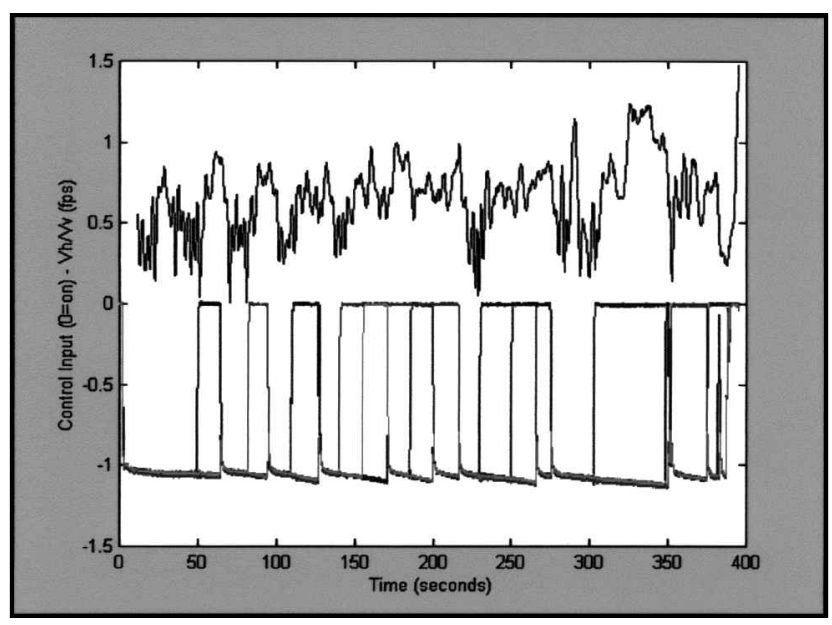

Fig. 14 System response.

Flight-Test Results

The control system is intended to affect a change in horizontal velocity. This is best demonstrated by assessing the glide ratio of the system with the winds removed. Figure 14 presents the glide ratio with the measured control inputs. The results show that a nominal glide ratio of 0.4 to 0.5 exists for the C- 9 parachute with no control inputs. Potential causes of this induced glide are motion induced by the oscillations, imperfections in length of the pressurized actuators, the mathematics of creating a horizontal glide ratio, which eliminates direction of motion or errors in the wind estimate. This nominal glide ratio does not limit the assessment of the response caused by control input, as we are interested in the change of glide ratio at the time of control activation. At time zero all PMAs were pressurized. The system was then allowed to stabilize to a "trim" condition. A change in glide ratio is apparent at approximately $20 \mathrm{~s}$ with no change in the state of the controls. The first incident of change in glide ratio can be attributed to the parachute inflation and stabilization process. The remaining data clearly show a correlation of glide ratio changes to the activation of the controls.

Figure 15 isolates the response of a single control input. An increase in glide ratio from approximately 0.5 to approximately 1.0 with a time constant of about 4 to $5 \mathrm{~s}$ is observed. The system 


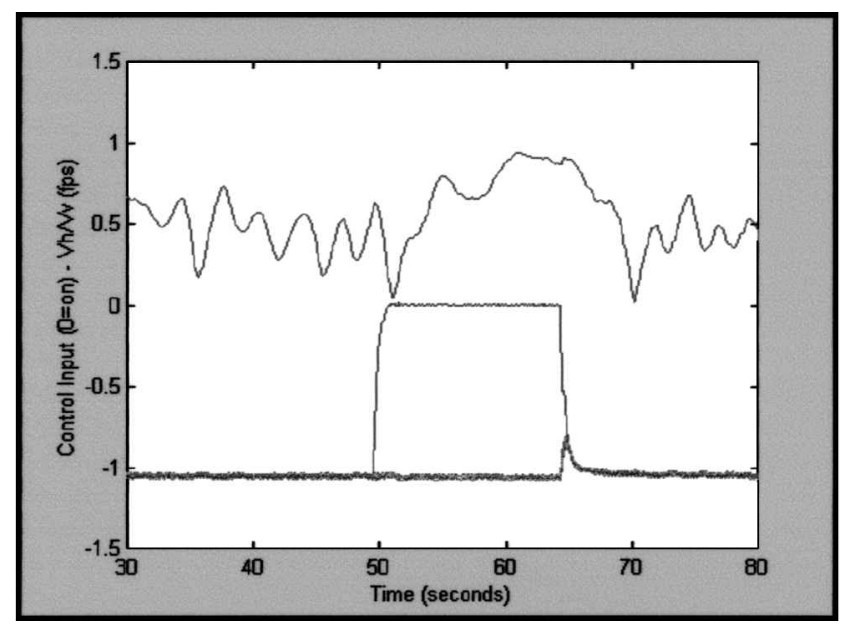

Fig. 15 Single control response.

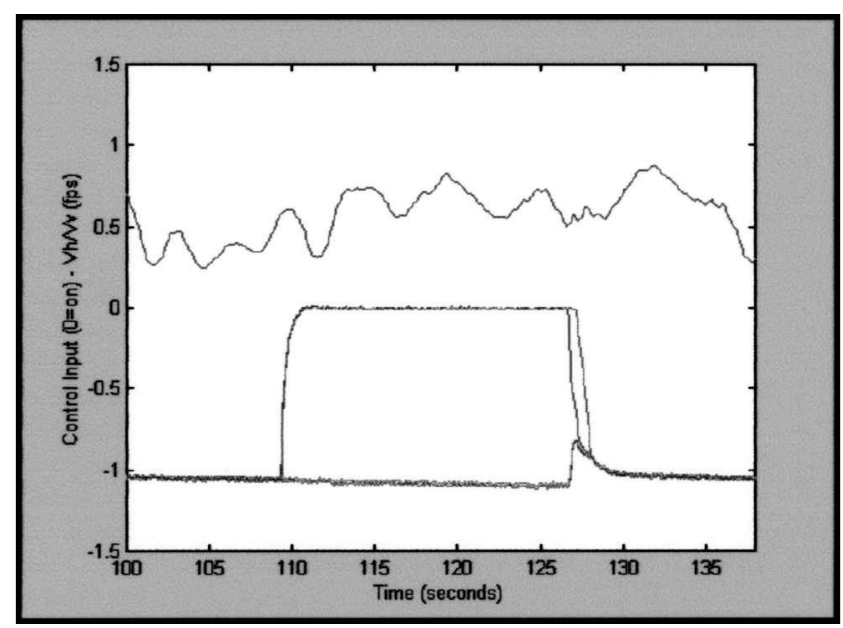

Fig. 16 Two control responses.

returns to its oscillatory trim state after about $5 \mathrm{~s}$ following removal of the control input. The reduced magnitude of oscillation or coning angle contributes to a reduced rate of descent and increased glide ratio. Recall that the two control inputs can be activated simultaneously. The intent is to provide additional resolution (every $45 \mathrm{deg}$ ) in controlling the system. Figure 16 presents the response with two simultaneous inputs.

As exemplified by this figure, there is no increase in performance with two control inputs over that achieved with one. In fact, the data indicate reduced response results from the simultaneous activation of two PMAs. This reduced performanceis likely caused by leadingedge collapse (as observed in flight test) of the parachute with two control inputs. The magnitude of the oscillations is not reduced as dramatically as with a single control input.

\section{Model Verification}

Figure 17 presents the measured velocity data from flight test as compared to the modeled velocity data for an uncontrolled drop. The "noise" in the measured data results from the velocity being measured at the payload, which is experiencing significant oscillations. Because the point-mass model does not incorporate these oscillations, no noise is apparent in the modeled data. As is demonstrated in the graph, the velocity data agree very well for this uncontrolled condition. For this run atmospheric density measurements were not available. Therefore, the modeled descent rate does not match precisely with the measured descent rate, but the differences appear negligible. Initializing the model at the start position of the

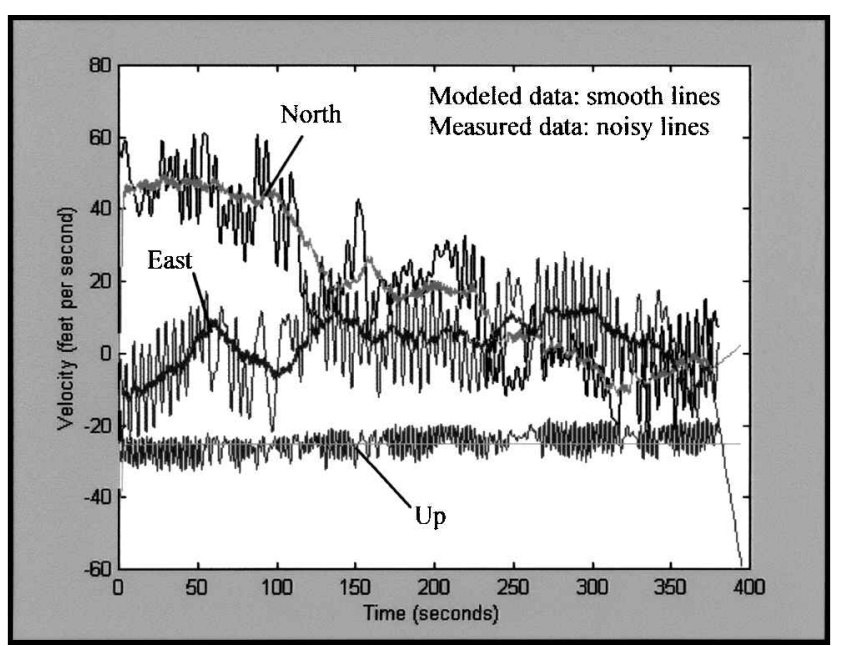

Fig. 17 Measured vs modeled velocity.

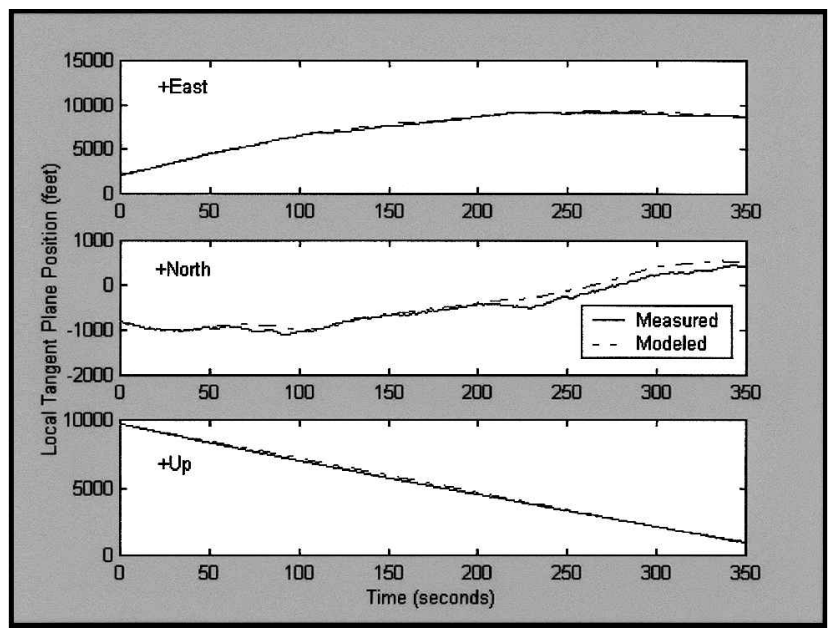

Fig. 18 Measured vs modeled position.

flight test, the model's ability to estimate position of the system was evaluated.

Figure 18 shows the accurate prediction of the flight path under the given wind conditions.

\section{Simulation}

The individualmodeling efforts have been presented. These models were integrated into a full system simulation using Simulink. ${ }^{\circledR}$ Figure 19 provides a block diagram of the overall simulation concept. To obtain the statistical base desired, Monte Carlo capabilities were added to the simulation. The parameters randomly selected during the simulation include 1) planned wind file (used to obtain predicted trajectory), 2) GPS selective availability errors included (or not) in the simulation, 3) offset from predicted release point, 4) parachute system turn rate, 5) release altitude, and 6) compass bias.

\section{Results}

Six-hundred simulations were conducted. ${ }^{10}$ These results include the achieved accuracy improvement of the controlled system over that of an uncontrolled system. The simulations produced excellent results with an accuracy of $210 \mathrm{ft}(64 \mathrm{~m})$ CEP. The total average horizontal error was $309 \mathrm{ft}(94 \mathrm{~m})$ with an average of 15 control inputs being required. The maximum number of control inputs for all 600 simulations was 33 . Figure 20 presents a threedimensional plot of several of the simulation results. This figure illustrates the initial release points and flight paths to impact with the 


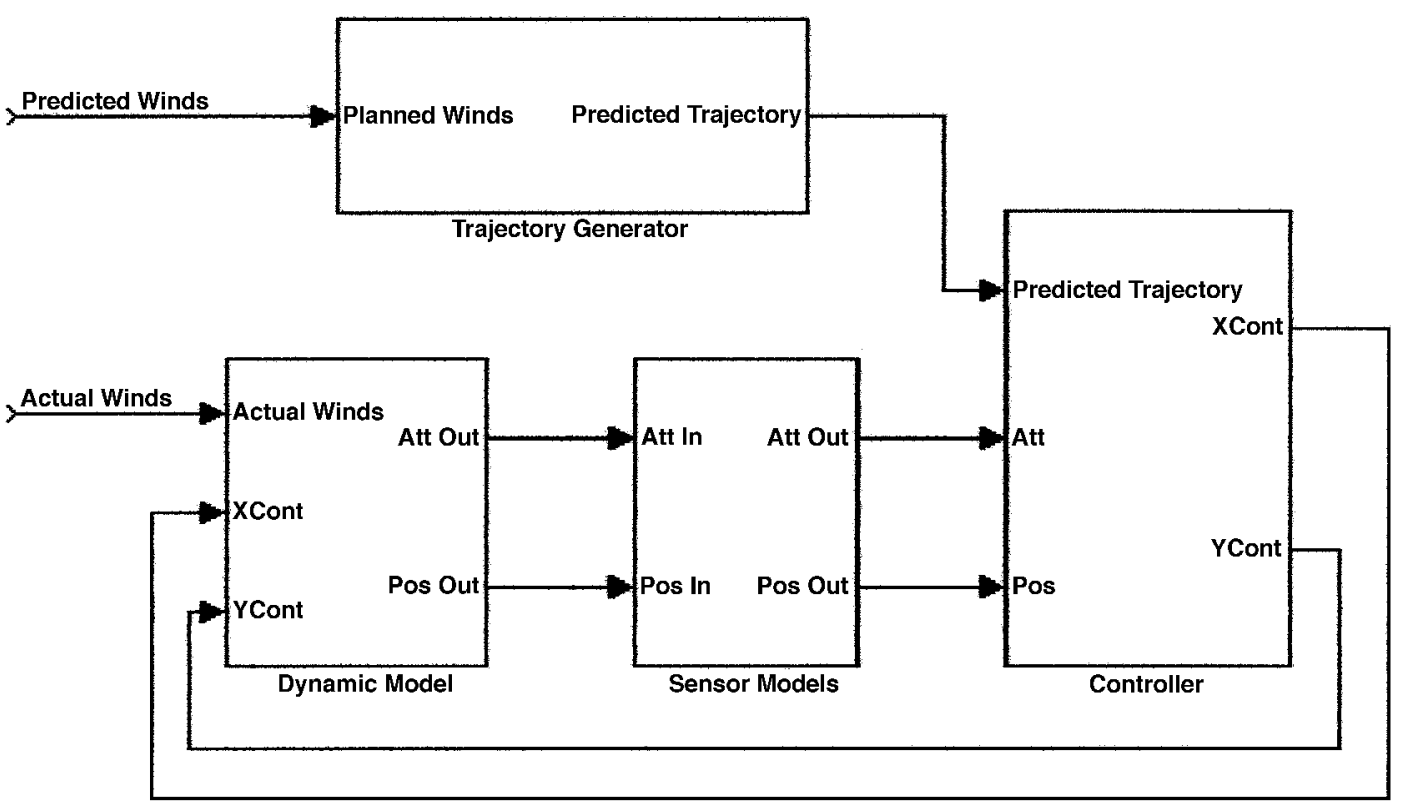

Fig. 19 Simulation overview.

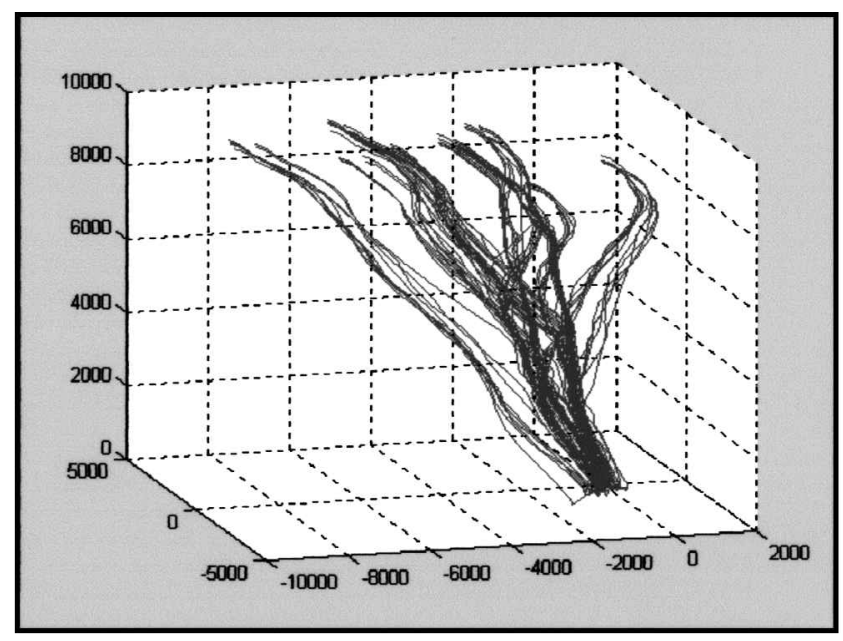

Fig. 20 Simulation results.

center of the coordinatesystem $(0,0)$ being the planned impact point. With the exception of a few trials, the system guided to the desired target.

The analysis of these results demonstrated that the predominant factor influencing accuracy of the control system was the time from the predicted winds used to establish the planned trajectory to that of the time of the simulated airdrop.

\section{Conclusions}

This study demonstrated that the AGAS concept has potential of providing a cost-effective option for precision airdrop. Simulations demonstrate that accuracies of $210 \mathrm{ft}(64 \mathrm{~m})$ CEP can be achieved. Additional efforts are needed to optimize the control system in an effort to further reduce the amount of stored gas required on the operationalsystem. The flight-test program provided flight dynamic data for the AGAS system. An efficient Monte Carlo type simulation was developed using a point-mass model for parachute dynamics, sensor models for GPS, and heading information, and a Bang-Bang type control system. The point-mass concept for system modeling is adequate for this evaluation. The position and velocity results from the model matched well with the measured flight-test results under the same wind conditions. However, to optimize the control system, a full six-degree-of-freedom model is likely required to capture the proper heading response.

Six-hundred simulations were conducted with randomly selected initialization parameters. These results demonstrated that the AGAS, as described herein, shows strong potential of providing a low-cost alternative for precision airdrop. Three critical factors will drive the final design of the AGAS. First, the accuracy of the estimated winds when determining the planned trajectory is the dominant factor in the accuracy of the AGAS concept. Wind data of up to six hours old (as compared to the "actual" winds used in the simulation) resulted in large horizontal errors from the desired impact points. Second, the rotation rate of the parachute system is important. Rotation rates with a mean of $1.89 \mathrm{deg} / \mathrm{s}$ and a standard deviation of $1 \mathrm{deg} / \mathrm{s}$ allowed effective control. If the rotation rates of the production system are increased from that, sufficient control may not be possible. Finally, the number of control inputs required (maximum of 33 inputs demonstrated in simulation) to achieve the desired accuracy is marginal under the current control concept. The prototype fuel system currently under design has been specified to allow for a maximum of 25 control inputs. There is insufficient fuel in the current design to ensure the accuracies can be met. However, no attempts were made to optimize the control algorithms for minimum fuel usage.

\section{Follow-On Work}

This study demonstrated the feasibility of the AGAS concept. However, the success of the final design rides on the three critical factors just presented. Therefore, the following recommendations are offered for follow-on work:

1) Complete the incorporation of six-degree-of-freedom equations of motion. Nonlinear parameter estimation techniques should be investigated to support this effort.

2) Fully characterize the performance of the AGAS concept using the G-12 and/or G-11 parachute systems. The remote-controlled activation technique used on the $\mathrm{C}-9$ test program should be applied to the G-12 system as soon as G-12 actuators are available.

3) Investigate optimizing the control algorithms for minimum fuel usage. The current methodology provides minimum horizontal errors without regard to fuel consumption.

\section{References}

1 "Summary Report: New World Vistas, Air and Space Power for the 21st Century," USAF Science Advisory Board, 1997. 
${ }^{2}$ Brown, G., Haggard, R., Almassy, R., Benney, R., and Dellicker, S., "The Affordable Guided Airdrop System," AIAA Paper 99-1742, June 1999.

${ }^{3}$ Brown, G., Haggard, R., Benney, R., and Rosato, N., "A New Pneumatic Actuator: Its Use in Airdrop Applications," AIAA Paper 99-1719, June 1999.

${ }^{4}$ Lamb, S. H., Hydrodynamics, 6th ed., Dover, New York, 1945.

${ }^{5}$ Doherr, K. F., and Saliaris, C., "On the Influence of Stochastic and Acceleration Dependent Aerodynamic Forces on the Dynamic Stability of Parachutes," AIAA Paper 81-1941, May 1981.

${ }^{6}$ Braasch, M. S., "A Signal Model for GPS," Journal of the Institute of
Navigation, Vol. 37, No. 4, 1990-1991.

${ }^{7}$ Kaminer, I., "Lecture Notes, AA3276 Introduction to Avionics," Naval Postgraduate School, Sept. 1997.

${ }^{8}$ System Identification User's Manual, The Mathworks, Inc., Natick, MA, 1997.

9 "Final Report: Development and Demonstration of a Ram-Air Parafoil Precision Guided Airdrop System, Vol. 3," Draper Lab., Army Contract DAAK60-94-C-0041, National Soldier Center, Oct. 1996.

${ }^{10}$ Dellicker, S., "Low Cost Parachute Guidance, Navigation, and Control," M.S. Thesis, Aeronautical and Astronautical Engineering, Naval Postgraduate School, Monterey, CA, Sept. 1999. 